\title{
REFLECTANCIA FOLIAR, CRECIMIENTO Y RESPUESTAS FISIOLÓGICAS DE MAÍZ Y TOMATE A UN ABRILLANTADOR ÓPTICO
}

\author{
FOLIAR REFLECTANCE, GROWTH AND PHYSIOLOGICAL RESPONSES OF MAIZE AND \\ TOMATO TO AN OPTICAL BRIGHTENER
}

\author{
Sandra P. Velasco, ${ }^{1,2}$ Samuel Pineda, ${ }^{1}$ Aricely Méndez,${ }^{1,2}$ Ma. Luisa España, ${ }^{1}$ Raúl Cárdenas-Navarro, ${ }^{1}$ \\ Jeannette S. Bayuelo-Jiménez, ${ }^{1}$ Javier Valle ${ }^{3}$, José I. Figueroa, Philippe Lobit ${ }^{1}$ y Ana M. Martínez \\ Castillo $^{1^{*}}$
}

\begin{abstract}
${ }^{1}$ Instituto de Investigaciones Agropecuarias y Forestales, Universidad Michoacana de San Nicolás de Hidalgo. Km. 9.5 Carretera Morelia-Zinapécuaro. 58880 Morelia, Michoacán, México. Tel. y Fax 01 (443) 29583 23. Facultad de Biología, Universidad Michoacana de San Nicolás de Hidalgo. 58030 , Morelia, Michoacán, México. ${ }^{3}$ Colegio de la Frontera Sur (ECOSUR). Apdo. Postal 36. Km. 2.5 carretera antiguo aeropuerto. 30700 Tapachula, Chiapas, México.

* Autor para correspondencia (amabel_66@hotmail.com)
\end{abstract}

\section{RESUMEN}

Se evaluó el efecto del abrillantador óptico Tinopal® C1101 (derivado del etenedil bencenosulfónico) a 1 y $3 \%(p / v)$ sobre la reflectancia foliar, crecimiento, fotosíntesis y conductancia estomática de dos cultivos agrícolas, maíz (Zea mays L.) y tomate (Solanum lycopersicum L.). La aplicación del abrillantador óptico sobre las hojas de maíz y tomate incrementó el porcentaje de reflectancia en un intervalo de 1.5 a 2.5 y de 1.6 a 1.9 veces, respectivamente, en las longitudes de onda correspondientes al espectro de absorción de las clorofilas $a$ y $b$. Su aplicación continua a 1 y $3 \%$ sobre plantas de maíz en condiciones de invernadero, afectó la altura de planta, área foliar $y$ peso seco $(P \leq \mathbf{0 . 0 5})$. En contraste, tales tratamientos no afectaron el crecimiento de las plantas de tomate. La aplicación del abrillantador a 1 y $3 \%$ no afectó la tasa de asimilación de $\mathrm{CO}_{2}$ ni la conductancia estomática de maíz y tomate. Estos resultados indican que el efecto de este abrillantador óptico sobre el crecimiento de las plantas puede estar relacionado con diferencias estructurales $o$ fisiológicas de las mismas.

Palabras clave: Zea mays, Solanum lycopersicum, abrillantadores ópticos, bioinsecticidas, crecimiento, fotosíntesis.

\section{SUMMARY}

The effects of the optical brightener Tinopal ${ }^{\circledR}$ C1101 (an ethenediyl benzenesulfonic derivative) at 1 and $3 \%(w / v)$ on the reflectance, growth, photosynthesis and stomatal conductance of two crops, maize (Zea mays L.) and tomato (Solanum lycopersicum L.), were evaluated. Application of the optical brightener increased reflectance on maize and tomato leaf surfaces ranging from 1.5 to 2.5 and 1.6 to 1.9 fold, respectively, in the wavelength regions corresponding to the absorption spectra of chlorophylls $a$ and $b$. Continuous application of Tinopal ${ }^{\circledR} \mathrm{C} 1101 \mathrm{1}$ and $3 \%$ on maize plants under greenhouse conditions, affected plant height, leaf area and dry weight $(P \leq \mathbf{0 . 0 5})$. In contrast, such treatments did not affect growth of tomato plants. The application of this brightener did not affect the photosynthetic rate nor stomatal conductance of maize and tomato. These results showed that the effects of this optical brightener on plant growth could be related with structural or physiological differences among plant species.

Index words: Zea mays, Solanum lycopersicum, optical brighteners, bioinsecticides, plant growth, photosynthesis.

\section{INTRODUCCIÓN}

Los baculovirus son un grupo de virus patógenos específicos para artrópodos, que en la actualidad se utilizan como bioinsecticidas en varios países del mundo (Szewezyk et al., 2006). No obstante, la radiación ultravioleta (UV) solar afecta la capacidad infectiva de las partículas virales y disminuye la eficacia de estos bioinsecticidas (Copping y Menn, 2000). Esto ha motivado la realización de estudios dirigidos a la identificación de sustancias que, incorporadas en los formulados bioinsecticidas, permitan incrementar su efectividad y persistencia en condiciones de campo (Martínez et al., 2005; Lasa et al., 2007). Al respecto, se han descrito los efectos de abrillantadores ópticos en las formulaciones de baculovirus (Shapiro, 1992; Okuno et al., 2003), los cuales son productos ampliamente utilizados en la elaboración de detergentes, plásticos y papel. Pertenecen a varios grupos químicos (ej., distirilbifenilo, estirilbenceno, oxaleno y estilbeno) que se caracterizan por absorber la luz UV y transmitirla en el espectro de luz visible (Argauer y Shapiro, 1997).

Los abrillantadores ópticos pueden proporcionar niveles altos de protección a los baculovirus expuestos a la radiación UV en condiciones de laboratorio o campo. Además, 
ciertos abrillantadores ópticos, como Tinopal LPW ${ }^{\circledR}$ (Sigma, St. Louis, MO, USA) (derivado del estilbeno), pueden incrementar la infectividad de estos patógenos contra sus huéspedes, particularmente en estadios tardíos (Shapiro y Argauer, 2001; Murillo et al., 2003; Martínez et al., 2003), y así incrementar los niveles de control de plagas (Lasa et al., 2007). Los abrillantadores ópticos se patentaron bajo la premisa de incrementar la posibilidad de éxito de diferentes microorganismos utilizados en el control biológico de insectos plaga (Shapiro et al., 1992). Sin embargo, existen pocos estudios en donde se haya evaluado el impacto de estos compuestos sobre el desarrollo de las plantas cultivadas. Un estudio bajo condiciones de invernadero mostró que aplicaciones foliares del Tinopal ${ }^{\circledR}$ CBS (derivado del diestiril bifenil) a 1 y $5 \%$ redujeron el desarrollo de plantas de maíz (Zea mays L.) y trigo (Triticum aestivum L.) en cerca de 25 y 30 a $40 \%$, respectivamente (Goulson et al., 2003). Adicionalmente, los autores observaron que las aplicaciones de Tinopal ${ }^{\circledR}$ CBS incrementaron la reflectancia foliar, particularmente en la región $420-470 \mathrm{~nm}$, lo cual quizás alteró el comportamiento de insectos polinizadores como Bombus spp. (Hymenoptera: Apidae) (Goulson et al., 2000).

El objetivo del presente estudio fue evaluar el efecto del abrillantador óptico Tinopal ${ }^{\circledR} \mathrm{C} 1101$ (etenedil bencenosulfónico) a 1 y $3 \%$ sobre la reflectancia foliar, crecimiento, fotosíntesis y conductancia estomática de dos cultivos de importancia agrícola. Dicho compuesto fue seleccionado debido a su potencial como fotoprotector de un formulado de baculovirus para el control de Spodoptera frugipera (J. E. Smith) (Lepidoptera: Noctuidae), la plaga más importante del maíz en México (Mondragón et al., 2007).

\section{MATERIALES Y MÉTODOS}

Las plantas utilizadas en el presente estudio se mantuvieron en un invernadero localizado en el municipio de Tarímbaro, Michoacán, ubicado a $19^{\circ} 46^{\prime} 116^{\prime \prime}$ LN y $101^{\circ} 08^{\prime} 966^{\prime \prime}$ LO y a una altitud de $1860 \mathrm{~m}$. Las especies evaluadas fueron maíz var. 'HY-311' y tomate (Solanum lycopersicum L.) var. 'Floradade IT'. La germinación de las semillas de maíz se llevó a cabo sobre papel húmedo y las de tomate en semilleros de plástico con celdas individuales de $3 \times 3 \mathrm{~cm}$ que contenían un sustrato rico en humus. Posteriormente, las plántulas de las dos especies se trasplantaron en una mezcla $(1: 3, \mathrm{v} / \mathrm{v})$ de fibra de coco y grava volcánica de óxido de hierro $(0.5-1.0 \mathrm{~cm})$ contenido en bolsas de plástico de color negro de 25 x 25 $\mathrm{cm}$. Las plantas se fertilizaron sólo una vez, al aplicar 0.5 $\mathrm{g}$ /planta de la fórmula 15N-20P-15K (Industrias Agrícolas Unidas, Zamora, México) a los 4 d (maíz) y 8 d (tomate) posteriores al trasplante. Los riegos se llevaron a cabo dos veces por semana con agua desmineralizada $(300 \mathrm{~mL}$ por planta). El abrillantador óptico Tinopal ${ }^{\circledR} \mathrm{C} 1101$ fue obtenido como muestra de laboratorio de Ciba Especialidades Químicas, México, D. F.

\section{Reflectancia de la superficie foliar}

Se determinó en tres hojas completamente expandidas seleccionadas al azar tanto en maíz como en tomate, que se sumergieron en una de las siguientes soluciones: i) Tinopal ${ }^{\circledR}$ C1101 a $1 \%+0.1 \mathrm{~g} \mathrm{~L}^{-1}$ del tensoactivo aniónico sodio dodecil sulfato (SDS); ii) Tinopal ${ }^{\circledR} \mathrm{C} 1101$ a $3 \%+0.1 \mathrm{~g} \mathrm{~L}^{-1}$ de SDS; y iii) $0.1 \mathrm{~g} \mathrm{~L}^{-1}$ de SDS a 0.01 $\%$ (testigo). Todas las soluciones se prepararon con agua destilada. En maíz se utilizó un fragmento de $\sim 4 \mathrm{~cm}$ de longitud de cada hoja. Se hicieron tres repeticiones por cada concentración. Posterior a la aplicación del tratamiento, las hojas se dejaron secar a temperatura ambiente durante $15 \mathrm{~min}$. El espectro de reflectancia de cada hoja (tomate) o fragmento de hoja (maíz) se midió con un espectrofotómetro Perkin Elmer ${ }^{\circledR}$ Lambda 35 UV/Vis Systems (Shelton, CT, USA) con esfera de integración con un intervalo de longitud de onda de 200 a $1100 \mathrm{~nm}$, donde la reflectancia se midió en tres posiciones diferentes.

Para examinar el porcentaje de reflectancia de las plantas de maíz y tomate expuestas a la radiación solar, ambas especies se asperjaron (con aspersor manual) con una solución que contenía Tinopal ${ }^{\circledR} \mathrm{C} 110$ a $3 \%$ o únicamente SDS como testigo. Para cada tratamiento se utilizaron seis plantas, mismas que fueron agrupadas para incrementar la cobertura foliar (Pellat et al., 2005). La aspersión de los tratamientos se hizo dentro del invernadero y, una vez que las soluciones se secaron, las plantas se transportaron cuidadosamente al exterior para ser expuestas a la luz solar. La reflectancia foliar se midió con un espectrofotómetro de campo (FieldSpec Pro ${ }^{\circledR}$, Analytical Spectral Devices, Boulder, CO, USA) con un intervalo de longitud de onda de 350 a $800 \mathrm{~nm}$. El espectro se midió en diez posiciones diferentes sobre cada grupo de plantas. Los valores de la reflectancia se calibraron en un panel de reflectancia Spectralon ${ }^{\circledR}$. Las medidas se tomaron a las 2, 72 y $168 \mathrm{~h}$ posteriores a la aplicación de los tratamientos entre las 09:00 y 12:00 h. Después de cada medición las plantas se regresaron al invernadero.

\section{Efecto del Tinopal ${ }^{\circledR}$ C1101 sobre el crecimiento y características fisiológicas del maíz y tomate}

Se utilizaron plantas de maíz y tomate de 19 y 30 d posteriores al trasplante, respectivamente. Las plantas se asperjaron con un aspersor manual que contenía una de 
las siguientes soluciones: Tinopal ${ }^{\circledR} \mathrm{C} 1101$ a 1 y $3 \%+$ $0.1 \mathrm{~g} \mathrm{~L}^{-1}$ de SDS, o únicamente SDS como testigo. Para cada tratamiento se utilizaron tres repeticiones de cinco plantas por especie, y las aspersiones se aplicaron una vez por semana durante cuatro semanas (Goulson et al., 2003). La posición de los tratamientos fue al azar en un área de $64 \mathrm{~m}^{2}$. Un día antes de cada aplicación se tomaron datos de altura y número de hojas. Posterior a la última evaluación, todas las plantas se cortaron desde su base para medir área foliar y peso seco total; para área foliar, todas las hojas de cada planta se cortaron y colocaron en un medidor de área foliar L1-3100® (LI-COR, Inc., USA). Para determinar el peso seco, las hojas y el tallo de cada planta se introdujeron en bolsas de papel aluminio y se colocaron en una estufa a $80{ }^{\circ} \mathrm{C}$ durante $2 \mathrm{~d}$. Todas las muestras se pesaron en una balanza digital (Denver XP-3000®, USA).

La tasa de asimilación de $\mathrm{CO}_{2}$ y la conductancia estomática en condiciones de invernadero, se midieron en plantas de maíz y tomate de 47 y 60 d después del trasplante, respectivamente. Estas plantas se asperjaron con la misma técnica y soluciones mencionadas anteriormente, a las 06:00 h. Tres horas después de la aplicación, se hicieron las mediciones sobre una hoja completamente expandida por planta, con un medidor de fotosíntesis portátil LI-6400® (LICOR, Inc., USA). Cada medición se repitió cinco veces con hojas de edad similar procedentes de plantas diferentes. La tasa neta de fotosíntesis se midió a una presión parcial externa de $\mathrm{CO}_{2}$ de $34 \mathrm{MPa}\left(340 \mu \mathrm{mol} \mathrm{CO} \mathrm{mol}^{-1}\right.$ aire $)$ y un déficit de presión de vapor del aire de $4.4 \mathrm{KPa}$. La densidad de flujo fotónico fue de 1400 y $2000 \mu \mathrm{mol} \mathrm{m}^{-2} \mathrm{~s}^{-1}$ para tomate y maíz, respectivamente, la cual se reguló artificialmente. Todas las mediciones se llevaron a cabo entre las 09:00 y 13:00 h en un día soleado.

\section{Análisis de los datos}

Los valores medios del porcentaje de reflectancia (procedentes del espectrofotómetro de laboratorio), peso seco, área foliar y fotosíntesis, fueron comparados entre tratamientos mediante un análisis de varianza. La comparación de medias se hizo con la prueba de diferencia mínima significativa (DMS, $\mathrm{P}<0.05)$ con el programa SPSS 12.0 (SPSS, Chicago, IL, USA). El porcentaje de reflectancia fue analizado sólo con los datos obtenidos de los picos correspondientes al espectro de absorción de las clorofilas $a(430$ y $660 \mathrm{~nm})$ y $b(460 \mathrm{y}$ $640 \mathrm{~nm}$ ) (Nobel, 1999). El espectro de reflectancia de las medidas obtenidas del espectrofotómetro de campo (Figuras 1 y 2) se expresó como reflectancia relativa, la cual se define como el cociente del porcentaje de reflectancia de las plantas tratadas con el Tinopal ${ }^{\circledR} \mathrm{C} 1101$ a $3 \%$ y las no tratadas (Blackmer et al., 1996).

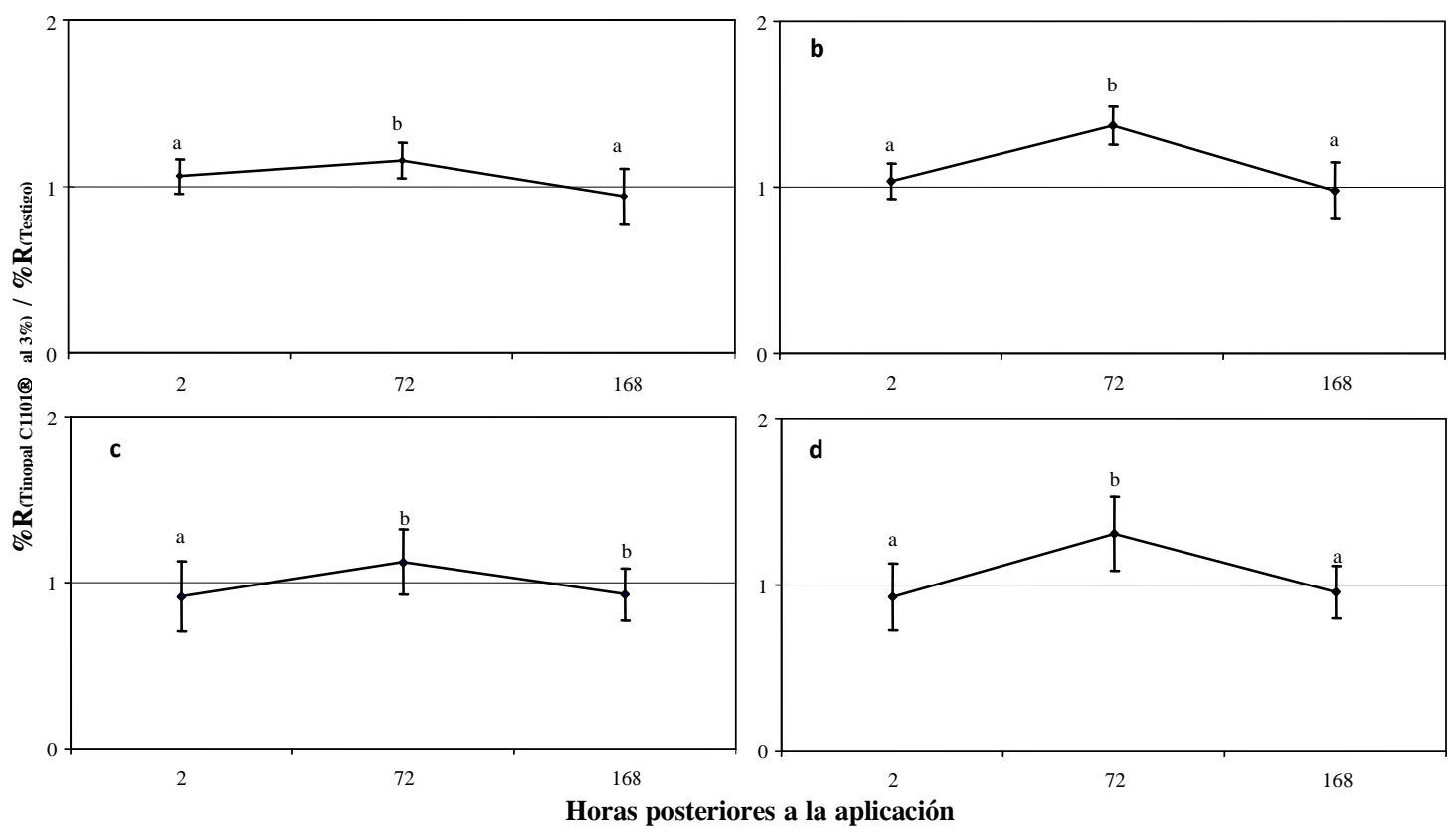

Figura 1. Promedio y error estándar de la relación (cociente) entre la reflectancia media (\%) de plantas de un dosel de maíz tratadas con Tinopal C1101® a $3 \%$ y la reflectancia media de plantas no tratadas (testigo), para las longitudes de onda 430 (a), 460 (b), 640 (c) y 660 (d) $\mathrm{nm}$, a 2,72 y 168 h después de la aplicación. Datos seguidos de la misma letra no son significativamente diferentes dentro de cada periodo de tiempo (prueba $t$ de Student, $P>0.05)$. 


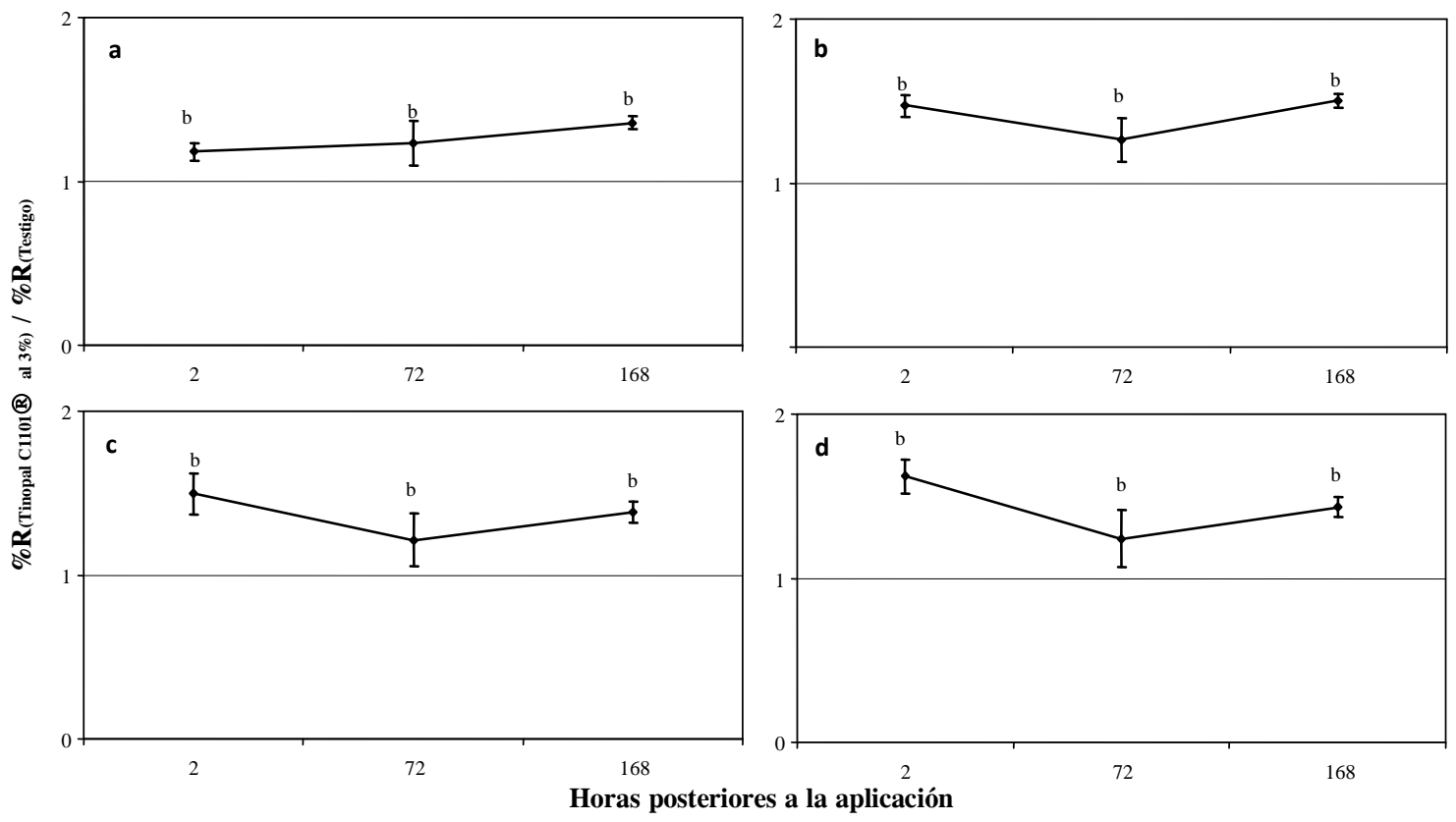

Figura 2. Promedio y error estándar de la relación (cociente) entre la reflectancia media (\%) de plantas de un dosel de tomate tratadas con Tinopal $\mathrm{C} 1101 @$ a $3 \%$ y la reflectancia media de plantas no tratadas (testigo), para las longitudes de onda 430 (a), 460 (b), 640 (c) y 660 (d) nm, a 2, 72 y 168 h después de la aplicación. Datos seguidos de la misma letra no son significativamente diferentes dentro de cada periodo de tiempo (prueba $t$ de Student, $P>0.05$ ).

La separación de las medias de reflectancia se hizo mediante la prueba $t$ de Student. La altura y número de hojas por planta se analizaron a través de modelos mixtos, en donde las especies y dosis se consideraron efectos fijos. La correlación entre las observaciones por cada planta a través del tiempo fue establecida por la correlación Toeplitz. La diferencia significativa entre tratamientos fue determinada mediante contrastes ortogonales. Estos últimos análisis se llevaron a cabo en el programa estadístico SAS (Statistical Analysys System., Inc., Cary, NC, USA).

\section{RESULTADOS}

\section{Reflectancia de la superficie foliar}

El porcentaje de reflectancia foliar de las hojas de maíz se incrementó $(\mathrm{P} \leq 0.01)$ en un intervalo de 1.5-2.5 veces por la aplicación del $\operatorname{Tinopal}^{\circledR} \mathrm{C} 1101$ a 1 y $3 \%$ en todas las longitudes de onda analizadas, en comparación con el testigo (Cuadro 1). En tomate, dicho incremento sólo fue significativo $(\mathrm{P} \leq 0.01)$ en la concentración más alta (3 \%), en un intervalo de 1.6-1.9 veces, y la concentración a $1 \%$ a una longitud de onda de $430 \mathrm{~nm}$.

Cuadro 1. Promedio de reflectancia $(\% \pm$ EE) a diferentes longitudes de onda en hojas de maíz y tomate tratadas con el abrillantador óptico Tinopal ${ }^{\circledR}$ C1101 a 1 y $3 \%(p / v)$.

\begin{tabular}{|c|c|c|c|c|}
\hline \multirow[b]{2}{*}{ Tratamientos } & \multicolumn{4}{|c|}{ Longitud de onda (nm) } \\
\hline & 430 & 460 & 640 & 660 \\
\hline \multicolumn{5}{|l|}{ Maíz } \\
\hline Testigo & $6.91 \pm 0.26 \mathrm{a}$ & $7.06 \pm 0.26 \mathrm{a}$ & $7.43 \pm 0.29 a$ & $6.35 \pm 0.24 \mathrm{a}$ \\
\hline Tinopal ${ }^{\circledR}$ C1101-1 \% & $13.20 \pm 0.63 b$ & $15.38 \pm 0.69 b$ & $11.53 \pm 0.57 b$ & $10.14 \pm 0.48 b$ \\
\hline Tinopal ${ }^{\circledR}$ C1101-3 \% & $14.52 \pm 0.70 b$ & $17.88 \pm 0.87 \mathrm{c}$ & $11.69 \pm 0.38 b$ & $10.23 \pm 0.38 b$ \\
\hline Valor de F & $51.49 * *$ & $73.81 * *$ & $30.94 * *$ & $33.45^{* *}$ \\
\hline \multicolumn{5}{|l|}{ Tomate } \\
\hline Testigo & $6.10 \pm 0.38 \mathrm{a}$ & $5.83 \pm 0.36 \mathrm{a}$ & $6.34 \pm 0.37 \mathrm{a}$ & $5.15 \pm 0.32 \mathrm{a}$ \\
\hline Tinopal ${ }^{\circledR}$ C1101-1 \% & $7.70 \pm 0.47 \mathrm{~b}$ & $6.86 \pm 0.44 \mathrm{a}$ & $6.53 \pm 0.35 \mathrm{a}$ & $5.47 \pm 0.32 \mathrm{a}$ \\
\hline Tinopal ${ }^{\circledR}$ C1101-3 \% & $11.84 \pm 0.86 \mathrm{c}$ & $11.11 \pm 0.89 \mathrm{~b}$ & $9.88 \pm 0.79 b$ & $8.66 \pm 0.68 b$ \\
\hline Valor de $\mathrm{F}$ & $23.45^{* *}$ & $20.58 * *$ & $13.39 * *$ & $16.96 * *$ \\
\hline
\end{tabular}

Medias con letras iguales en cada columna no son significativamente diferentes entre sí (DMS, 0.05).

** Significativo a una probabilidad de 0.01 . 
En las plantas de maíz tratadas con Tinopal ${ }^{\circledR}$ C1101 a 3 $\%$, tal incremento del porcentaje de reflectancia foliar comparado con el testigo solamente ocurrió a las $72 \mathrm{~h}$ posteriores a la aplicación (Figura 1), mientras que a las $168 \mathrm{~h}$ hubo una disminución $(\mathrm{P} \leq 0.02)$ del porcentaje de reflectancia en longitud de onda de $640 \mathrm{~nm}$. En contraste, la aplicación del Tinopal ${ }^{\circledR}$ C1101 a $3 \%$ sobre las plantas de tomate provocó un incremento $(\mathrm{P} \leq 0.01)$ de la reflectancia en un intervalo de 1.18-1.64 veces en todas las longitudes de onda y en los tres periodos de tiempo analizados (2, 72 y 168 h) en comparación con el testigo (Figura 2).

\section{Efecto del Tinopal@ C1101 sobre el crecimiento y otras características fisiológicas del maíz y tomate}

En las dos especies evaluadas, la altura de planta $\left(\mathrm{F}_{4,176}\right.$ $=294.2, \mathrm{P}<0.0001)$ y número de hojas $\left(\mathrm{F}_{4,176}=\right.$ 234.3, $\mathrm{P}<0.0001)$ se incrementaron durante el curso del experimento. En relación con el desarrollo de las mismas, se observó que las plantas de maíz tratadas con el abrillantador fueron más pequeñas $(\mathrm{P} \leq 0.05)$ a partir de la segunda semana de aplicado el tratamiento, en proporciones 14.4 a $16.4 \%$ y de 15.7 a $23.5 \%$ para las dosis de 1 y $3 \%$, respectivamente, comparadas con el testigo (Figura 3a). Las plantas de maíz también mostraron una tendencia a disminuir su número de hojas por efecto del abrillantador, pero el efecto no fue significativo comparado con el testigo $\left(\mathrm{F}_{2,87}=1.67, \mathrm{P}=\right.$ 0.194) (Figura 3b). En cuanto al peso de las plantas de maíz, el abrillantador óptico causó una reducción de 49 y $31 \%$ para las concentraciones 1 y $3 \%$, respectivamente, comparado con el testigo (8.6 g) (Cuadro 2). En el área foliar también se registraron reducciones de 36 y $54 \%$ cuando las plantas se trataron con el abrillantador a 1 y 3 $\%$, respectivamente, comparado con el testigo (568.8 $\mathrm{cm}^{2}$ ). La aplicación del Tinopal® C1101 al no afectó significativamente la tasa de asimilación de $\mathrm{CO}_{2}$ ni la conductancia estomática del maíz, comparadas con el testigo.
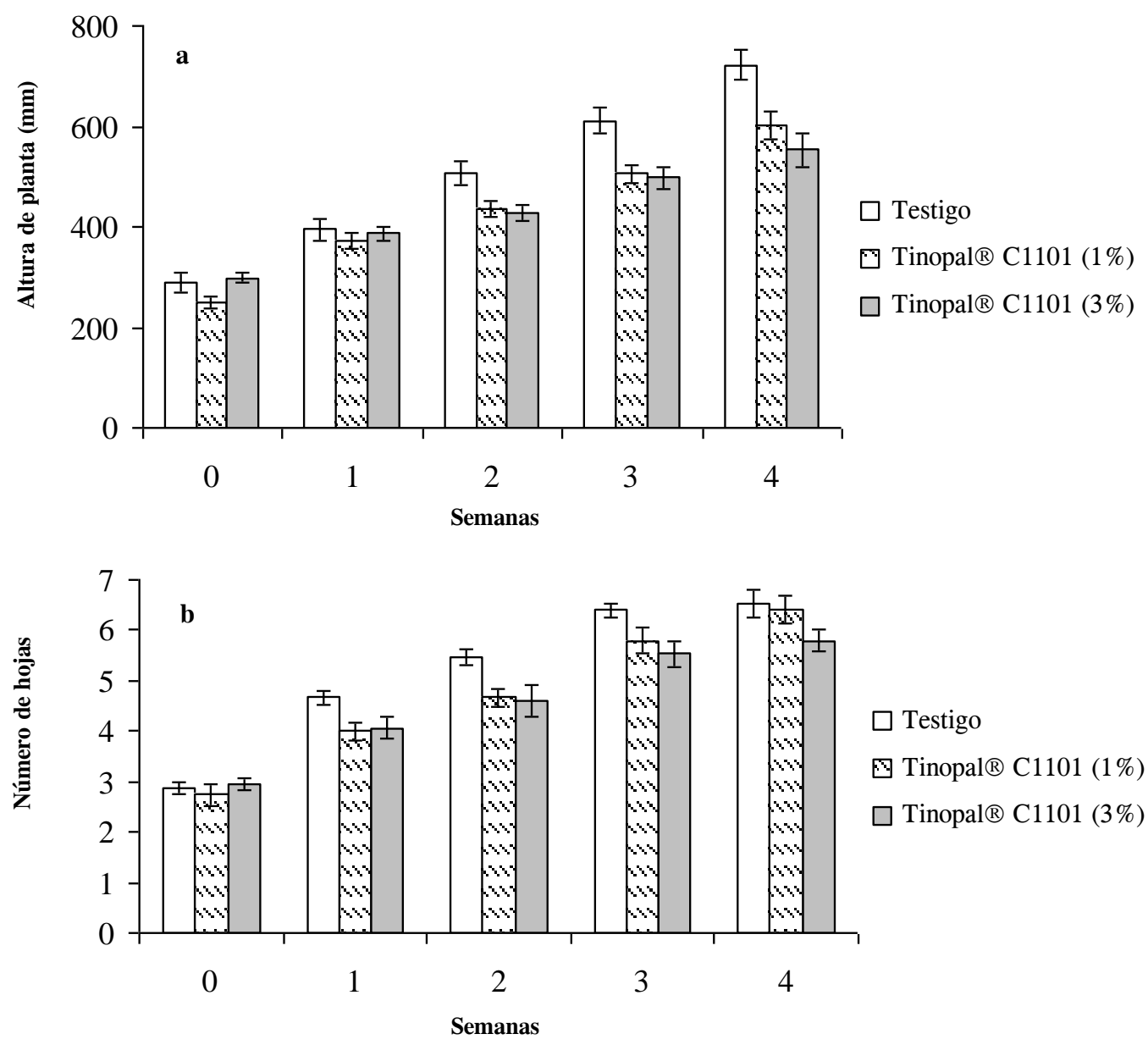

Figura 3. Promedio y error estándar de altura de planta $(\mathrm{mm} \pm$ ) (a) y número de hojas por planta (b) de plantas de maíz tratadas con el abrillantador óptico Tinopal C1101@ a 1 y $3 \%$, en un periodo de 4 semanas $(\mathrm{n}=15$ por tratamiento) (Contrastes ortogonales, $\mathrm{P}<0.05)$. 
Cuadro 2. Peso seco, área foliar, conductancia estomática y tasa de asimilación de $\mathrm{CO}_{2}$ de plantas de maíz y tomate tratadas con el abrillantador óptico Tinopal® C1101 a 1 y $3 \%$ (p/v). Promedio \pm error estándar.

\begin{tabular}{|c|c|c|c|c|c|}
\hline \multicolumn{2}{|c|}{ Tratamientos } & $\begin{array}{l}\text { Peso seco } \\
\text { (g) }\end{array}$ & $\begin{array}{l}\text { Área foliar } \\
\left(\mathrm{cm}^{2}\right)\end{array}$ & $\begin{array}{l}\text { Conductancia } \\
\text { estomática } \\
\left(\mathrm{mol} \mathrm{m}^{-2} \mathrm{~s}^{-1}\right)\end{array}$ & $\begin{array}{c}\text { Tasa de asimilación } \\
\text { de } \mathrm{CO}_{2} \\
\left(\mu \mathrm{molCO}_{2} \mathrm{~m}^{-2} \mathrm{~s}^{-1}\right)\end{array}$ \\
\hline \multirow{4}{*}{ Maíz } & Testigo & $8.56 \pm 1.57 \mathrm{a}$ & $568.83 \pm 79.32 \mathrm{a}$ & $0.09 \pm 0.01 \mathrm{a}$ & $19.68 \pm 2.48 \mathrm{a}$ \\
\hline & Tinopal ${ }^{\circledR}$ C1101 (1 \%) & $4.36 \pm 0.36 \mathrm{~b}$ & $364.07 \pm 55.75 \mathrm{~b}$ & $0.08 \pm 0.01 \mathrm{a}$ & $18.98 \pm 1.47 \mathrm{a}$ \\
\hline & Tinopal ${ }^{\circledR}$ C1101 (3 \%) & $5.92 \pm 0.73 b$ & $263.90 \pm 59.76 b$ & $0.11 \pm 0.02 \mathrm{a}$ & $25.01 \pm 1.68 \mathrm{a}$ \\
\hline & Valores de F & $4.28 *$ & $7.56^{* *}$ & $3.83 \mathrm{~ns}$ & $2.62 \mathrm{~ns}$ \\
\hline \multirow[t]{4}{*}{ Tomate } & Testigo & $4.66 \pm 0.51 \mathrm{a}$ & $286.50 \pm 28.78 \mathrm{a}$ & $0.20 \pm 0.06 \mathrm{a}$ & $16.02 \pm 1.59 \mathrm{a}$ \\
\hline & Tinopal ${ }^{\circledR}$ C1101 (1 \%) & $4.44 \pm 0.42 \mathrm{a}$ & $320.90 \pm 38.59 \mathrm{a}$ & $0.14 \pm 0.04 \mathrm{a}$ & $14.98 \pm 1.22 \mathrm{a}$ \\
\hline & Tinopal® C1101 (3 \%) & $3.71 \pm 0.34 \mathrm{a}$ & $258.60 \pm 22.30 \mathrm{a}$ & $0.20 \pm 0.05 \mathrm{a}$ & $17.92 \pm 1.47 \mathrm{a}$ \\
\hline & Valores de F & $1.31 \mathrm{~ns}$ & $1.02 \mathrm{~ns}$ & $0 . \overline{3} 1 \mathrm{~ns}$ & $1.08 \mathrm{~ns}$ \\
\hline
\end{tabular}

Medias con letras iguales en cada columna no son estadísticamente diferentes en sí (DMS, 0.05 ). *, ** Significativo a una probabilidad de 0.05 y 0.01 , respectivamente; ns $=$ no significativo.

Los resultados obtenidos en las plantas de tomate indicaron que el Tinopal ${ }^{\circledR}$ C1101 a 1 y $3 \%$ no afectó (P $\leq$ 0.05) la altura de planta y número de hojas durante curso del experimento (Cuadro 2). Los promedios de altura fluctuaron de $71.9 \pm 4.1$ a $216.4 \pm 6.6 \mathrm{~mm}$ para las plantas tratadas con el abrillantador, y de $76.2 \pm 7.9$ a $194.5 \pm 8.1 \mathrm{~mm}$ para las plantas testigo. El número de hojas fluctuó de $5.3 \pm 0.37$ a $10.1 \pm 0.63$ hojas en las plantas tratadas con el abrillantador y de $5.3 \pm 0.31$ a 9.1 \pm 0.51 hojas en las plantas testigo. Tampoco hubo diferencias significativas en área foliar, peso seco, tasa de asimilación de $\mathrm{CO}_{2}$ y conductancia estomática, en comparación con el testigo.

\section{DISCUSIÓN}

En el presente estudio la aplicación del abrillantador óptico Tinopal ${ }^{\circledR} \mathrm{C} 1101$ causó un incremento del nivel de reflectancia foliar del maíz y tomate en las longitudes de onda correspondientes al espectro de absorción de las clorofilas $a$ y $b$. Similarmente, un experimento efectuado en el Reino Unido indicó que una aplicación de Tinopal ${ }^{\circledR}$ CBS a 1 y $5 \%$ incrementó la reflectancia foliar de plantas de col (Brassica oleracea L.), particularmente en la región de 420 a $700 \mathrm{~nm}$ (Goulson et al., 2003).

El espectro de reflectancia foliar puede depender de la morfología de la planta, la concentración de pigmentos, la estructura celular y el contenido de agua (Pellat et al., 2005), aunque los niveles de reflectancia de la luz visible son generalmente bajos, cercanos a 6-10 \% de la luz incidente (Tang, 1997), lo cual es congruente con los niveles obtenidos en este estudio en las hojas testigo de maíz (6.3-7.4\%) y tomate (5.1-6.3\%), como se mostró en el Cuadro 1. La luz visible es indispensable para la regulación de la fotosíntesis y el movimiento de los estomas (Tang, 1997). Las clorofilas $a$ y $b$ son responsables de la absorción de la luz para la fotosíntesis y se encuentran en todas las plantas superiores (Nobel, 1999); por tanto, un incremento en la reflectancia de la luz en los picos de absorción de ambos pigmentos, provocado por los abrillantadores ópticos, podría tener un efecto negativo en la cantidad de luz disponible para dicho proceso.

Una interrogante planteada en este estudio fue determinar si las aplicaciones del abrillantador óptico Tinopal ${ }^{\circledR}$ C1101 sobre las hojas alterarían la tasa de asimilación de $\mathrm{CO}_{2}$ y la conductancia estomática. Los resultados mostraron que una aplicación del compuesto no afectó a las especies aquí evaluadas. No obstante, se observaron efectos negativos en el crecimiento de las plantas de maíz continuamente expuestas al Tinopal ${ }^{\circledR}$ C1101, pero no a las plantas de tomate. Estos resultados coinciden con los estudios de Lasa et al. (2007) quienes determinaron que la aplicación de tres concentraciones $(0.1,1.0$ y $5.0 \%)$ del abrillantador Leucophor AP (un derivado del estilbeno disulfonato), no afectó adversamente el desarrollo del pimiento (Capsicum annum L.) durante un periodo de $14 \mathrm{~d}$. Goulson et al. (2003) observaron que el Tinopal ${ }^{\circledR}$ CBS a 1 y $5 \%$ redujo la tasa de desarrollo de dos especies monocotiledóneas, maíz y cebada (Hordeum vulgare L.) en un rango de 25 a $40 \%$, pero no afectó el de especies dicotiledóneas, tales como col (Brassica oleracea L.) y haba (Vicia faba L.).

Lo anterior indica que el efecto de los abrillantadores ópticos sobre el desarrollo de las plantas puede estar determinado mayoritariamente por diferencias entre especies, como los patrones de desarrollo, la arquitectura o las propiedades químicas (Goulson et al., 2003), más que por diferencias estructurales entre los compuestos. $\mathrm{Al}$ respecto, es altamente probable que en plantas con hojas orientadas verticalmente, como las de maíz, las continuas aplicaciones de abrillantadores ópticos incrementarían la reflectancia de ambos lados de la hoja y así afectarían más la cantidad de luz utilizada para la fotosíntesis, en comparación con plantas de hojas orientadas horizontalmente como están en varias especies dicotiledóneas. Estos resultados pueden también reflejar diferentes respuestas a 
la intensidad de la luz en función del mecanismo fotosintético. En plantas tipo $\mathrm{C}_{4}$ como maíz, la tasa fotosintética se incrementa con la intensidad luminosa y no muestran un punto de saturación por luz (Usuda, 1987), mientras que en plantas $C_{3}$ como tomate, ocurre saturación por luz entre 1200 y $1400 \mu \mathrm{mol} \mathrm{m}^{-2} \mathrm{~s}^{-1}$ (Ayari et al., 2000). Por tanto, una disminución en la intensidad de la luz provocada por los abrillantadores ópticos podría reducir drásticamente la tasa fotosintética en plantas $\mathrm{C}_{4}$ comparadas con plantas $\mathrm{C}_{3}$.

Nótese que la tasa de asimilación de $\mathrm{CO}_{2}$ y conductancia estomática medidas en este estudio fueron menores que las observadas por Melkonian et al. (2004) y Ying et al. 2002) para maíz $\left[0.15 \mathrm{~mol} \mathrm{~m} \mathrm{~m}^{-2} \mathrm{~s}^{-1}\right.$ (conductancia estomática) y $43.2 \mu \mathrm{mol} \quad \mathrm{CO}_{2} \quad \mathrm{~m}^{2} \mathrm{~s}^{-1}$ (fotosíntesis), respectivamente] y Bradford et al. (1983) para tomate [0.37-0.91 mol m $\mathrm{m} \mathrm{s}^{-1}$ (conductancia estomática) y 21-24 $\mu \mathrm{mol} \mathrm{CO} 2 \mathrm{~m}^{2} \mathrm{~s}^{-1}$ (fotosíntesis)]. Tales diferencias podrían estar relacionadas con la edad de las plantas o las condiciones de luz utilizadas.

Se ha demostrado que algunos abrillantadores ópticos pueden ser altamente persistentes en el ambiente (Webb et $a l .$, 1994). Aunque en el presente estudio los residuos del Tinopal ${ }^{\circledR} \mathrm{C} 1101$ a 3\% no fueron medidos, el incremento de la reflectancia detectado en las plantas de tomate a las $168 \mathrm{~h}$ después de la aplicación sugiere la presencia de este compuesto sobre la superficie foliar. En maíz, el incremento de la reflectancia sólo se detectó hasta $72 \mathrm{~h}$ después de la aplicación. Las posibles diferencias en persistencia podrían reflejar una variación en las características químicas o físicas, como recubrimiento con ceras y pubescencia de las plantas sobre las cuales se aplicaron los abrillantadores ópticos (Vail et al., 1999).

En este estudio se observó que a las 72 y 168 h posteriores a la aplicación, las hojas de maíz estuvieron más abiertas, comparadas con las plantas analizadas a las $2 \mathrm{~h}$, lo cual pudo incrementar su exposición a la radiación solar y como consecuencia su reflectancia. Aún así, a las $168 \mathrm{~h}$ no se observaron diferencias entre las plantas tratadas con el abrillantador óptico y las del testigo, en tres $(430,460$ y $660 \mathrm{~nm})$ de las cuatro longitudes de onda evaluadas. Esto sugiere que la tasa de desarrollo causó una dilución de los residuos sobre las hojas, o que la presencia de hojas nuevas no tratadas influyó en los niveles de reflectancia.

Puesto que cualquier efecto negativo de los abrillantadores ópticos sobre las plantas cultivadas es indeseable para su producción, se recomienda que previo a la utilización masiva de los abrillantadores ópticos en condiciones de campo, se lleven a cabo estudios dirigidos a la evaluación de su impacto ambiental.

\section{CONCLUSIONES}

La aplicación del abrillantador óptico Tinopal ${ }^{\circledR}$ C1101 a 1 ó $3 \%$ sobre las plantas de tomate y maíz provocó un incremento en el porcentaje de reflectancia foliar, sin causarles efectos negativos sobre su conductancia estomática y fotosíntesis. Cuando el abrillantador óptico se aplicó continuamente sobre las plantas de maíz y tomate, únicamente la especie monocotiledónea fue afectada negativamente en su crecimiento.

\section{AGRADECIMIENTOS}

Al Programa de Mejoramiento del Profesorado-SEP (PROMEP-PTC-110) y a la Coordinación de la Investigación Científica (Proyecto 6.17) de la Universidad Michoacana de San Nicolás de Hidalgo.

\section{BIBLIOGRAFÍA}

Argauer R, M Shapiro (1997) Fluorescence and relative activities of stilbene optical brighteners as enhancers for the gypsy moth (Lepidoptera: Lymantriidae) baculovirus. J. Econ. Entomol. 90:416-420.

Ayari O, G Samson, M Dorais, R Boulanger, A Gosselin (2000) Stomatal limitation of photosynthesis in winter production of greenhouse tomato plants. Physiol. Plant. 110:558-564.

Blackmer T M, J S Schepers, G E Varvel, E A Walter-Shea (1996) Nitrogen deficiency detection using reflected shortwave radiation from irrigated corn canopies. Agron. J. 88:1-5.

Bradford K J, T D Sharkey, G D Farquhar (1983) Gas exchange, stomatal behavior, and $\delta^{13} \mathrm{C}$ values of the flacca tomato mutant in relation to the abscisic acid. Plant Physiol. 72:245250.

Copping L G, J J Menn (2000) Biopesticides: a review of their action applications and efficacy. Pest Manage. Sci. 56:651676.

Goulson D, L C Derwent, D I Penagos, T Williams (2003) Effects of optical brighteners included in biopesticide formulations on the growth of crops. Agric. Ecosyst. Environ. 95:235-240.

Goulson D, A M Martínez, O H Hughe, T Williams (2000) Effects of optical brighteners used in biopesticide formulations on the behaviour of pollinators. Biol. Control 19:232-236.

Lasa R, C Ruíz-Portero, M D Alcázar, J E Belda, P Caballero, T Williams (2007) Efficacy of optical brightener formulations of Spodoptera exigua multiple nucleopolyhedrovirus (SeMNPV) as a biological insecticide in greenhouses in southern Spain. Biol. Control 40:89-96.

Martínez A M, O Simón, T Williams, P Caballero (2003) Effect of optical brighteners on the insecticidal activity of a nucleopolyhedrovirus in three instars of Spodoptera frugiperda (Lepidoptera: Noctuidae). Entomol. Exp. Appl. 109:139-146.

Martínez A M, T Williams, M López-Ferber, P Caballero (2005) Optical brighteners do not influence covert baculovirus infection of Spodoptera frugiperda. Appl. Environ. Microbiol. 71:1668-1670.

Melkonian J, L X Yu, T L Setter (2004) Chilling responses of maize (Zea mays L.) seedlings: root hydraulic conductance, abscisic acid, and stomatal conductance. J. of Exp. Bot. 55:1751- 
1760.

Mondragón G, S Pineda, A Martínez, A M Martínez (2007) Optical brightener Tinopal C1101 as an ultraviolet protectant for a nucleopolyhedrovirus. Comm. Agric. Appl. Biol. Sci. 72:243-247.

Murillo R, R Lasa, D Goulson, T Williams, D Muñoz, P Caballero (2003) Effect of Tinopal LPW on the insecticidal properties and genetic stability of the nucleopolyhedrovirus of Spodoptera exigua (Lepidoptera: Noctuidae). J. Econ. Entomol. 96:1668-1674.

Nobel P S (1999) Physicochemical and enviromental plant physiology. Academic Press, San Diego, USA. 474 p.

Okuno S, J Takatsuka, M Nakai, S Ototake, A Masui, Y Kunimi (2003) Viral-enhancing activity of various stilbene-derived brighteners for a Spodoptera litura (Lepidoptera: Noctuidae) nucleopolyhedrovirus. Biol. Control 26:146-152.

Pellat F P, E Palacios, E Mejía, M Martínez, L A Palacios (2005) Análisis de los espacios espectrales de la reflectividad del follaje de cultivos. Agrociencia 39:293-301.

Shapiro M (1992) Use of optical brighteners as radiation protectants for gypsy moth (Lepidoptera: Lymantriidae) nuclear polyhedrosis virus. J. Econ. Entomol. 85:1682-1686.

Shapiro M, R Argauer (2001) Relative effectiveness of selected stilbene optical brighteners as enhancers of the beet armyworm (Lepidoptera: Noctuidae) nuclear polyhedrosis virus. J. Econ. Entomol. 94:339-343.
Shapiro M, E M Dougherty, J J Hamm (1992) Compositions and methods for biocontrol using fluorescent brighteners. U. S. Patent No. 5,124,149. Disponible en: www.patentstorm.us/ patents/ /5124149/ (Octubre, 2009).

Szewezyk B, L H Carvajal, M Paluszek, I Skreccz, M Lobo de Souza (2006) Baculovirus; re-emerging biopesticides. Biotechno. Adv. 24:143-160.

Tang Y (1997) Light. In: Plant Ecophysiology. M N V Prasad (ed). John Wiley \& Sons Inc. New York, USA. pp:3-40.

Usuda $\mathbf{H}$ (1987) Changes in levels of intermediates of the $\mathrm{C}_{4}$ cycle and reductive pentose phosphate pathway under various light intensities in maize leaves. Plant Physiol. 84:549-554.

Vail P V, F Darlene, F Hoffmann, J S Tebbets (1999) Influence of fluorescent brighteners on the filed activity of the celery looper nucleopolyhedrovirus. Southwestern Entomol. 24:8798.

Webb R E, M Shapiro, J D Podwaite, R L Ridgway, L Venables, G B White, R J Argauer, D L Cohen, J Witcosky, K M Kester, K W Thorpe (1994) Effect of optical brighteners on the efficacy of gypsy moth (Lepidoptera: Lymantriidae) nuclear polyhedrosis virus in forest plots with high or low levels of natural virus. J. Econ. Entomol. 87:134-143.

Ying J, E A Lee, M Tollenaar (2002) Response of leaf photosynthesis during the grain-filling period of maize to duration of cold exposure, acclimation, and incident PPFD. Crop Sci. 42:1164-1172. 